\title{
ON FLAT FAMILIES OF FAT POINTS
}

\author{
GIUSEPPE PAXIA
}

(Communicated by Louis J. Ratliff, Jr.)

\begin{abstract}
The aim of this paper is to fill a gap in a proof of a theorem by B. Segre regarding the regularity of linear systems of curves in $P^{2}$. This is done by showing that some subschemes of "fat points" of $P^{2}$ form a flat family over an integral quasiprojective variety of which we give an explicit construction.
\end{abstract}

\section{INTRODUCTION}

Many authors in various contexts have studied some zero-dimensional subschemes of the projective plane $\mathbb{P}_{k}^{2}=\mathbb{P}^{2}$ over an algebraically closed field $k$, which are usually known as subschemes of "fat points."

Given $s$ distinct points $\mathfrak{P}=\left(P_{1}, \ldots, P_{s}\right)$ and $s$ integers $\mathfrak{D}=\left(d_{1}, \ldots, d_{s}\right)$, we say subscheme of fat points relative to $\mathfrak{P}$ and $\mathfrak{D}$, the subscheme $Z(\mathfrak{P}, \mathfrak{D})$ defined by the homogeneous ideal $I=\mathfrak{p}_{1}^{d_{1}} \cap \cdots \cap \mathfrak{p}_{s}^{d_{s}}$, where $\mathfrak{p}_{i} \subseteq k\left[x_{0}, x_{1}, x_{2}\right]$ is the ideal corresponding to $P_{i}$. In such a way we get a zero-dimensional subscheme of $\mathbb{P}^{2}$ of degree $n=\sum n_{i}$, where $n_{i}=d_{i}\left(d_{i}+1\right) / 2$.

Let $S_{t}$ be the linear system consisting of all curves of degree $t$ containing $Z(\mathfrak{P}, \mathfrak{D})$ as a subscheme and $\mathfrak{J} \subseteq \mathbb{O}_{\mathbb{P}^{2}}$ be the ideal sheaf of $Z(\mathfrak{P}, \mathfrak{D})$. Then $S_{t}$ corresponds to $H^{0}\left(\mathbb{P}^{2}, \mathfrak{J}(t)\right)$.

The linear system is said to be regular if $H^{1}\left(\mathbb{P}^{2}, \mathfrak{J}(t)\right)=0$; this is equivalent to saying that $S_{t}$ has the "right" dimension.

It is a classical problem to decide when a linear system $S_{t}$ is regular but, since this is a hard problem in the general case, it is also interesting to find upper bounds for the integer

$$
\tau(Z(\mathfrak{P}, \mathfrak{D}))=\tau:=\min \left\{t \in \mathbb{N}: S_{t} \text { is regular }\right\} .
$$

B. Segre in [S] proves that for "generic" points $P_{1}, \ldots, P_{s}$ of $\mathbb{P}^{2}$, the linear system $S_{t}$ is regular for $t \geq \max \left\{d_{1}+d_{2}-1,\left[\sum d_{i} / 2\right]\right\}$, where $d_{1} \geq d_{2} \geq \cdots \geq$ $d_{s}$.

Received by the editors December 20, 1989.

1980 Mathematics Subject Classification (1985 Revision). Primary 14C05; Secondary 14H10.

This paper was written with the financial support of M.P.I. The author is a member of the G.N.S.A.G.A. of the C.N.R. 
Segre's proof consists essentially of two steps:

(a) He proves the theorem when the points lie on an irreducible conic.

(b) He deduces the theorem in general, admitting implicitly that the bound for the regularity cannot be decreased by "specializing" the generic given points to points on an irreducible conic.

But, indeed, statement (b) is not adequately justified, and cannot even be considered so obvious. The present article is an outcome of our attempts to fill up the gap in Segre's proof.

Our main result (Theorem 1) states that the schemes $Z(\mathfrak{P}, \mathfrak{D})$, for fixed $\mathfrak{D}$, move in a flat irreducible family, of which we give an explicit construction.

Theorem 1 can have many applications just by using known results. For example, it follows easily (by semicontinuity) that $\tau$ is an upper semicontinuous function of $\mathfrak{P}$ (Proposition 1), and this allows us to fix Segre's proof. Moreover, we can get information on the Betti numbers of the schemes $Z(\mathfrak{P}, \mathfrak{D})$ and on their locus in Hilb $^{n} \mathbb{P}^{2}$ (Corollary 2).

\section{THE MAIN ReSUlT}

For terminology and details not explicitly mentioned one can see [Se], [H1], [H2].

Theorem 1. For any s-tuple of fixed positive integers $\mathfrak{D}=\left(d_{1}, \ldots, d_{s}\right)$, there is a flat family $p: X \rightarrow T$, where $T$ is a smooth irreducible quasiprojective variety, parametrizing all the schemes of fat points $Z(\mathfrak{P}, \mathfrak{D})$, for all $\mathfrak{P}$ 's.

Proof. Consider the closed set $\Delta \subseteq\left(\mathbb{P}^{2}\right)^{s}$ whose closed points are $\left(P_{1}, \ldots, P_{s}\right)$ with $P_{i}=P_{j}$ for some $i \neq j$, and let $T$ be the complement of $\Delta$. Then let $\Delta_{i}$, for $i=1, \ldots, s$, be the closed subset of $T \times \mathbb{P}^{2}$ whose closed points are $\left(P_{1}, \ldots, P_{s}, P\right)$, with $P=P_{i}$.

Let $\mathfrak{J}_{i}$ be the ideal sheaf of $\Delta_{i}$ considered as a reduced subscheme of $T \times \mathbb{P}^{2}$. Finally, let $X$ be the subscheme of $T \times \mathbb{P}^{2}$ corresponding to the ideal sheaf $\mathfrak{J}=\mathfrak{J}_{1}^{d_{1}} \cap \cdots \cap \mathfrak{J}_{s}^{d_{s}}$.

In such a way we get a projective family

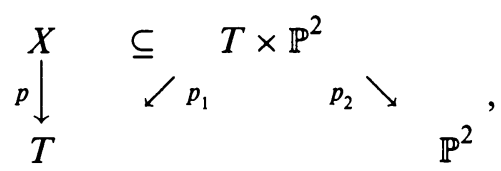

where $p$ is the restriction of the projection $p_{1}$.

We want to show that $p: X \rightarrow T$ is the required family. If we fix affine coordinates $\left(x_{i}, y_{i}\right)$ in a standard affine open subset $U_{i}$ of the $i$ th component of $\left(\mathbb{P}^{2}\right)^{s}$ and $(x, y)$ in a standard affine open subset of the ambient space $\mathbb{P}^{2}$, one has that in $U_{i}$ the ideal of $\mathfrak{J}_{i}$ is given locally by $\left(x_{i}-x, y_{i}-y\right)$. We have to verify that if $t=\left(P_{1}, \ldots, P_{s}\right) \in T$, then

$$
p_{2}\left(p^{-1}(t)\right)=Z\left(\mathfrak{p}_{1}^{d_{1}} \cap \cdots \cap p_{s}^{d_{s}}\right),
$$


that is, the subscheme of $\mathbb{P}^{2}$ associated to the ideal $I=\mathfrak{p}_{1}^{d_{1}} \cap \cdots \cap \mathfrak{p}_{s}^{d_{s}}$. That means that one can "read" at once in the ambient space $\mathbb{P}^{2}$ the subscheme of the considered fat points with the due multiplicities $d_{1}, \ldots, d_{s}$.

On the affine charts of $\left(\mathbb{P}^{2}\right)^{s}$, the ideal of $t$ is

$$
\mathfrak{a}=\left(x_{1}-a_{1}, y_{1}-b_{1}, \ldots, y_{s}-b_{s}\right),
$$

where $\left(a_{i}, b_{i}\right)$ are the coordinates of $P_{i}$ in $U_{i}$. On the considered charts the fiber $p^{-1}(t)$ is

$$
\operatorname{Spec}(R / \mathfrak{a} R),
$$

where $R=k\left[x_{i}, y_{i}, x, y\right] / \bar{I}, \bar{I}$ is the ideal of sections of $\mathfrak{J}$ on the affine open set where we are working, and $\mathfrak{a} R$ is obtained by extending $a$ by the canonical homomorphism $k\left[x_{i}, y_{i}\right] \rightarrow R$.

By a direct computation, we have

$$
\begin{aligned}
R / \mathfrak{a} R= & k\left[x_{i}, y_{i}, x, y\right] / \bar{I}+\left(x_{1}-a_{1}, \ldots, y_{s}-b_{s}\right) \\
= & k\left[x_{i}, y_{i}, x, y\right] /\left(x_{1}-a_{1}, \ldots, y_{s}-b_{s}\right) / \\
& \quad \bar{I}+\left(x_{1}-a_{1}, \ldots, y_{s}-b_{s}\right) /\left(x_{1}-a_{1}, \ldots, y_{s}-b_{s}\right) \\
& =k[x, y] /\left(x-a_{1}, y-b_{1}\right)^{d_{1}} \cap \cdots \cap\left(x-a_{s}, y-b_{s}\right)^{d_{s}},
\end{aligned}
$$

and this proves $(1)$.

Our family $p: X \rightarrow T$ is obviously flat, since the Hilbert polynomial of the fiber $p^{-1}(t)$ is constant (see [H1, Theorem 9.9]), and moreover by construction one has that $T$ is an integral quasiprojective smooth variety.

Remark 1. It is clear that the same proof (only with a more complicated notation) allows us to prove the theorem also for schemes of fat points in $\mathbb{P}^{r}$, for any $r$.

\section{CONSEQUences}

We start this section by recalling some known results. In [B-G, Proposition 1.7] is proved the following:

Proposition 1. Let $f: X \rightarrow Y$ be a flat family of projective schemes, where $Y$ is integral and Noetherian. Then the set $V=\left\{y \in Y: H\left(X_{y}, n\right)\right.$ is maximal for all $n$ \} is open and nonempty.

As usual, $H\left(X_{y}, \cdot\right)$ means the Hilbert function of the fiber $X_{y}$. As we said in the introduction, for $\mathfrak{P}$ and $\mathfrak{D}$ fixed, one can define the integer $\tau(\mathfrak{P}, \mathfrak{D})=$ $\min \left\{t \in \mathbb{N}: S_{t}\right.$ is regular $\}$.

We consider now $\mathfrak{D}$ fixed; as we proved in Theorem 1 , there is a flat irreducible family $p: X \rightarrow T$, parametrizing all the subschemes of fat points $Z(\mathfrak{P}, \mathfrak{D})$, for all $\mathfrak{P}$ 's. We can consider the function $\tau: T \rightarrow \mathbb{N}$, defined as

$$
\tau(t)=\min \left\{l \in \mathbb{N}: H\left(X_{t}, m\right)=n, \text { for } m \geq l\right\},
$$

where $n=\sum d_{i}\left(d_{i}+1\right) / 2$.

Clearly, for $\bar{t}$ fixed, one has $\tau(\bar{t})=\tau(\overline{\mathfrak{P}}, \mathfrak{D})$ where $\overline{\mathfrak{P}}$ is the set of $s$ distinct 
points corresponding to $\bar{t}$. The following proposition holds:

Proposition 2. The function $\tau$ is "upper semicontinuous" on $T$. The subset $V^{\tau} \subseteq T$ where $\tau$ is minimal is hence a nonempty open set such that $V \subseteq V^{\tau}$. Proof. For any $t \in T$ and fixed $n$, there exists an open set $U_{n} \ni t$ such that, for all $t^{\prime} \in U_{n}$, one has $H\left(X_{t^{\prime}}, n\right) \geq H\left(X_{t}, n\right)$ by Grauert's semicontinuity theorem (see, e.g., [B-G, Proposition 1.5] for details). For $t \in T$ consider $\tau(t)=n_{0}$. Then there exists $U_{n_{0}} \ni t$ such that $H\left(X_{t^{\prime}}, n_{0}\right) \geq H\left(X_{t}, n_{0}\right)=n$, for all $t^{\prime} \in U_{n_{0}}$. Hence $\tau(t)$ is upper semicontinuous on $T$. From this it follows that the subset $V^{\tau} \subseteq T$, where $\tau$ is minimal, is a nonempty open set. Since the parameter space $T$ is irreducible, it follows that $V^{\tau} \cap V \neq \varnothing$ and, $\tau$ being constant on $V$, we have $V \subseteq V^{\tau}$.

Remark 2. From the previous result, it follows that the gap in the proof of Segre's theorem is filled up, provided that by "generic points" of $\mathbb{P}^{2}$ one means a set of $s$ distinct points $\mathfrak{P} \in V^{\tau}$.

Remark 3. By using [B-G] and Theorem 1 one can get informations on the Betti numbers of the schemes of fat points. For example (see [B-G, Proposition 2.15]), we have the following:

Corollary 1. If $t$ varies in $V$, the Betti numbers of $Z(\mathfrak{P}, \mathfrak{D})$ are lower semicontinuous.

Now let $H=$ Hilb $^{n} \mathbb{P}^{2}$ be the Hilbert scheme parametrizing the zero-dimensional subschemes of $\mathbb{P}^{2}$ of degree $n$, and let $H \rightarrow H$ be the tautological family. It is known that the following universal property holds: for any flat family $S \rightarrow T$ of zero-dimensional subschemes of $\mathbb{P}^{2}$ of degree $n$, there exists a unique morphism $\phi: T \rightarrow H$ for which the diagram

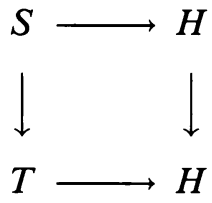

is commutative and cartesian. For details see, e.g., [Se].

From diagram (D) we get a morphism $\phi: T \rightarrow H$. It is interesting to understand the nature of $\phi(T)$ inside $H$. The best we can state is the following:

Corollary 2. Let $H=\operatorname{Hilb}^{n} \mathbb{P}^{2}$, where $n=\sum d_{i}\left(d_{i}+1\right) / 2$, and let $H(\mathfrak{D})=\phi(T)$ be the set of points of $H$ corresponding to the schemes $Z(\mathfrak{P}, \mathfrak{D})$. Then

(1) $H(\mathfrak{D})$ is constructible.

(2) If $s=1$, then $H(\mathfrak{D})$ is closed.

Proof. (1) From the diagram (D), $\phi: T \rightarrow H$ is a morphism of finite type of Noetherian schemes; so, (1) is a consequence of Chevalley's theorem (see [H1, Example 3.19]).

(2) In this case $T=\mathbb{P}^{2}$, and since $H$ is proper, then $\phi(T)$ is closed. 
Remark 4. Many seem to be sure that $\phi(T)$ is a locally closed subset of $H$ but, to the best of our knowledge, no proof of this fact exists in the literature. It would be interesting to know more about this.

\section{REFERENCES}

[B-G] M. Boratinsky and S. Greco, Hilbert functions and Betti numbers in a flat family, Ann. Mat. Pura Appl. (4) 142 (1985), 277-292.

[H1] R. Hartshorne, Algebraic geometry, Graduate Texts in Math., vol. 52, Springer, New York and Berlin, 1977.

[H2] J. Harris, Curves in the projective space, Sem. Math. Sup., Presses Univ. Montréal, Montreal, PQ, 1982.

[S] B. Segre, Alcune questioni su insiemi finiti di punti in geometria algebrica, Atti Convegno Intern. di Torino, 1961.

[Se] E. Sernesi, Topics on families of projective schemes, Queen's Papers in Pure and Appl. Math., vol. 73, Queen's Univ., Kingston, ON, 1986.

Dipartimento di Matematica, universita di Catania, Viale A. Doria, 6 Catania, Italy 